Article

\title{
Timing Performance Simulation for 3D 4H-SiC Detector
}

\author{
Yuhang Tan ${ }^{1,2}$, Tao Yang ${ }^{1,2}$, Kai Liu ${ }^{1}$ (D), Congcong Wang ${ }^{1}$, Xiyuan Zhang ${ }^{1}$, Mei Zhao ${ }^{1}$, Xiaochuan Xia ${ }^{3}$, \\ Hongwei Liang ${ }^{3}$, Ruiliang $X u^{3}{ }^{\text {, Yu Zhao }}{ }^{4}$, Xiaoshen Kang ${ }^{4}$, Chenxi Fu ${ }^{5}$, Weimin Song ${ }^{5} \mathbb{D}$, Zhenzhong Zhang ${ }^{3}$, \\ Ruirui Fan ${ }^{1,6}{ }^{\mathbb{D}}$, Xinbo Zou ${ }^{7}$ (D) and Xin Shi ${ }^{1, *(\mathbb{D})}$
}

1 Institute of High Energy Physics, Chinese Academy of Sciences, Beijing 100049, China; tanyuhang@ihep.ac.cn (Y.T.); yangtao@ihep.ac.cn (T.Y.); liukai@ihep.ac.cn (K.L.); wangcc@ihep.ac.cn (C.W.); zhangxiyuan@ihep.ac.cn (X.Z.); zhaomei@ihep.ac.cn (M.Z.); fanrr@ihep.ac.cn (R.F.)

2 School of Physical Sciences, University of Chinese Academy of Sciences, Beijing 100049, China

3 School of Microelectronics, Dalian University of Technology, Dalian 116024, China; xiaochuan@dlut.edu.cn (X.X.); hwliang@dlut.edu.cn (H.L.); xrl@mail.dlut.edu.cn (R.X.); zhangzz@dlut.edu.cn (Z.Z.)

4 School of Physics, Liaoning University, Shenyang 110036, China; yuzhao@ihep.ac.cn (Y.Z.); kangxiaoshen@lnu.edu.cn (X.K.)

5 College of Physics, Jilin University, Changchun 130012, China; fucx1619@mails.jlu.edu.cn (C.F.); weiminsong@jlu.edu.cn (W.S.)

6 Spallation Neutron Source Science Center, Dongguan 523803, China

7 School of Information Science and Technology, ShanghaiTech University, Shanghai 201210, China; zouxb@shanghaitech.edu.cn

* Correspondence: shixin@ihep.ac.cn

check for updates

Citation: Tan, Y.; Yang, T.; Liu, K.; Wang, C.; Zhang, X.; Zhao, M.; Xia, X.; Liang, H.; Xu, R.; Zhao, Y.; et al. Timing Performance Simulation for 3D 4H-SiC Detector. Micromachines 2022, 13, 46. https://doi.org/ $10.3390 / \mathrm{mi} 13010046$

Academic Editor: Francesco La Via

Received: 30 November 2021

Accepted: 23 December 2021

Published: 28 December 2021

Publisher's Note: MDPI stays neutral with regard to jurisdictional claims in published maps and institutional affiliations.

Copyright: (C) 2021 by the authors. Licensee MDPI, Basel, Switzerland. This article is an open access article distributed under the terms and conditions of the Creative Commons Attribution (CC BY) license (https:// creativecommons.org/licenses/by/ $4.0 /)$.

\begin{abstract}
To meet the high radiation challenge for detectors in future high-energy physics, a novel 3D $4 \mathrm{H}-\mathrm{SiC}$ detector was investigated. Three-dimensional $4 \mathrm{H}-\mathrm{SiC}$ detectors could potentially operate in a harsh radiation and room-temperature environment because of its high thermal conductivity and high atomic displacement threshold energy. Its 3D structure, which decouples the thickness and the distance between electrodes, further improves the timing performance and the radiation hardness of the detector. We developed a simulation software-RASER (RAdiation SEmiconductoR) — to simulate the time resolution of planar and 3D 4H-SiC detectors with different parameters and structures, and the reliability of the software was verified by comparing the simulated and measured time-resolution results of the same detector. The rough time resolution of the 3D $4 \mathrm{H}-\mathrm{SiC}$ detector was estimated, and the simulation parameters could be used as guideline to $3 \mathrm{D} 4 \mathrm{H}-\mathrm{SiC}$ detector design and optimization.
\end{abstract}

Keywords: 3D 4H-SiC detector; time resolution; RASER

\section{Introduction}

The main challenges of detectors close to the beam for future high-energy colliders lie in maintaining a high time and/or spatial resolution after large irradiation fluence and the requirement of a complex cooling system to sustain a low-temperature environment and to ensure these detectors work properly. The integrated irradiation fluence of future colliders like the High-Luminosity Large Hadron Collider (HL-LHC) will exceed $2 \times 10^{16} n_{e q} / \mathrm{cm}^{2}$ [1-3]. The timing performance of silicon detectors will deteriorate drastically under such high irradiation fluence. On the one hand, irradiation will decrease charge collection and degrade timing performance. For example, the charge collection of Low Gain Avalanche Detectors (LGAD) is reduced from around $35 \mathrm{fC}$ to $4 \mathrm{fC}$ after $3 \times 10^{15} n_{e q} / \mathrm{cm}^{2}$ irradiation, and the time resolution of the detector is difficult to be less than 30 ps under higher irradiation fluence. On the other hand, a complex cooling system is required to maintain the operating temperature of the detector at $-35^{\circ} \mathrm{C}[4,5]$. It is of great value to develop a time-resolution detector that is resistant to radiation and that can operate at room temperature.

One possible device to solve these challenges is a 3D silicon carbide ( $\mathrm{SiC}$ ) detector. 3D is a reliable technology to develop radiation hardness detectors [6,7], and $\mathrm{SiC}$ materials have the potential to work at room temperature or higher [8-10]. 
The 3D structure decouples the thickness of detector and the distance between electrodes. It can reduce the drift time of charge carriers and increase the deposited energy in the detector simultaneously. Irradiation mainly deteriorates the performance of the detector by generating defects that will trap the charge carrier and reduce the collection efficiency. The 3D structure may withstand higher irradiation fluence than a planar structure by improving the signal-to-noise ratio and reducing the probability of carriers being trapped by defects [11]. A 3D silicon pixel detector has been used in high-energy particle physics for precise position measurements, such as ATLAS Insertable B-Layer [12] and CMS-TOTEM Precision Proton Spectrometer [13], because of its superior radiation hardness. The 3D silicon pixel detector does not show significant degradation after $1 \times 10^{16} n_{e q} / \mathrm{cm}^{2}$ irradiation [7], and a 3D silicon strip detector still has a relative charge collection efficiency of $70 \%$ after $2 \times 10^{16} n_{e q} / \mathrm{cm}^{2}$ irradiation [14].

The $4 \mathrm{H}$ silicon carbide $(4 \mathrm{H}-\mathrm{SiC})$ has a high atomic displacement threshold energy, which can decrease the defects generated by irradiation, and $4 \mathrm{H}-\mathrm{SiC}$ is then potentially thought of as radiation-resistant material [15]. The high thermal conductivity of $4 \mathrm{H}-\mathrm{SiC}$ can easily control the temperature by being next to or in contact with the electronics. The saturated electron velocity of $4 \mathrm{H}-\mathrm{SiC}$ at $300 \mathrm{~K}$ is $2 \times 10^{7} \mathrm{~cm} \cdot \mathrm{s}^{-1}$, close to twice as much as silicon [9]. This will reduce the drift time, reduce the carrier trapping effect, and enhance time response sensitivity, which improves the radiation hardness and the timing performance of detector. The $4 \mathrm{H}-\mathrm{SiC}$ with a large bandgap energy has a low leakage current even under a high electric field, which is necessary for low-noise operation $[15,16]$. The 3D $4 \mathrm{H}-\mathrm{SiC}$ further reduces the drift time, improves the signal-to-noise ratio, and enhances the radiation resistance and timing performance of the detector.

Before irradiation, the current time resolution of the $3 \mathrm{D}$ silicon detector is about 75 ps [17], and the 3D-trench silicon is about 27 ps [18]. The 3D 4H-SiC detectors are expected to have a similar time resolution around $25 \mathrm{ps}$ before irradiation and a better time resolution after harsh irradiation comparing with the 3D silicon detector. The 3D 4H-SiC detector is one of the most promising device types to be a radiation-resistant high-precision time resolution detector that operates at room temperature.

Two main existing software programs could predict the time resolution of semiconductor detectors-KDetSim [19] and Weightfield2 [20]. KDetSim can estimate the hit-position contribution to the time resolution of 3D and planar silicon detectors. Weightfield2 mainly simulates the time resolution of planar silicon or diamond detectors. There is a lack of appropriate time-resolution-simulation software for 3D 4H-SiC detectors.

To estimate the timing performance of 3D $4 \mathrm{H}-\mathrm{SiC}$ detectors, we developed a fast simulation software-RASER (RAdiation SEmiconductoR) [21]. The electric field and weighting potential are calculated by FEniCS [22]. According to the time-resolution measurement $\beta$-setup [23,24], we simulated the tracks of two electrons with the same energy as electrons from ${ }^{90} \mathrm{Sr}$ source and the deposited energy in detectors with the max step length less than $1 \mu \mathrm{m}$ by GEANT4 [25]. The current induced by electron-hole (e-h) pairs moving is calculated by Shockley-Ramo's theorem [26]. The readout electronics used a simplified current amplifier [20].

We validated RASER by comparing measured and simulated time-resolution results of planar $4 \mathrm{H}-\mathrm{SiC}$ detectors [24]. The planar $4 \mathrm{H}-\mathrm{SiC}$ detectors are designed by Nanjing University (NJU), and the cross section of the detector is shown in Figure 1. The size of the detector is $5 \mathrm{~mm} \times 5 \mathrm{~mm}$, and the upper and lower electrodes are ohmic contacts. The detector has a $100 \mu \mathrm{m}$ high resistance active $4 \mathrm{H}-\mathrm{SiC}$ epitaxial layer and a $350 \mu \mathrm{m}$ substrate. Using the same parameters, the time resolution of measurement and simulation are $(83 \pm 1)$ ps and $(77 \pm 2)$ ps, respectively. In this study, we simulated the time resolution of $3 \mathrm{D} 4 \mathrm{H}-\mathrm{SiC}$ detectors with various structures and parameters, and the results would serve as a guideline for 3D $4 \mathrm{H}-\mathrm{SiC}$ detector design and optimization. 


\begin{tabular}{|c|c|c|}
\hline \multirow{2}{*}{$\begin{array}{l}\mathrm{Au} \\
1 \mu \mathrm{m}\end{array}$} & \multirow[b]{2}{*}{ Passivation layer } & \multirow{2}{*}{$\begin{array}{l}\mathrm{Au} \\
1 \mu \mathrm{m}\end{array}$} \\
\hline & & \\
\hline \multicolumn{3}{|c|}{$\mathrm{Ni} 75 \mathrm{~nm}$} \\
\hline \multicolumn{3}{|c|}{$\operatorname{Imp} \mathrm{P}^{+}$} \\
\hline \multicolumn{3}{|c|}{$100 \mu \mathrm{m} \mathrm{N} \mathrm{N}^{-} 4 \mathrm{H}-\mathrm{SiC}$ epi } \\
\hline \multicolumn{3}{|c|}{$350 \mu \mathrm{m} \mathrm{N} \mathrm{N}^{+} 4 \mathrm{H}-\mathrm{SiC}$ sub } \\
\hline & $\begin{array}{c}\mathrm{Ni} 75 \mathrm{~nm} \\
\mathrm{Ti} / \mathrm{Al} / \mathrm{Au} 1.5 \mu \mathrm{m}\end{array}$ & \\
\hline
\end{tabular}

Figure 1. Cross section of planar 4H-SiC detector designed by NJU.

\section{Detector and RASER}

\section{1. $3 \mathrm{D} 4 \mathrm{H}$-SiC Structure}

The structure of our 3D $4 \mathrm{H}-\mathrm{SiC}$ detector prototype is shown in Figure 2a, where the $p^{+}$electrode column that penetrates the entire high-resistance substrate perpendicular to the detector surface is surrounded by six $n^{+}$electrode columns. The intention of designing this structure is to obtain a more uniform electric field in the area surrounded by the $n^{+}$ electrodes. The simulation size of the 3D $4 \mathrm{H}-\mathrm{SiC}$ detector is $1 \mathrm{~cm} \times 1 \mathrm{~cm}$, and the thickness is $350 \mu \mathrm{m}$, and the effective concentration of n-type substrate is set to $1 \times 10^{13} \mathrm{~cm}^{-3}$. The radius of electrode column is $50 \mu \mathrm{m}$, and the column spacing, which is the distance between the center of $n^{+}$and $p^{+}$electrode columns is $150 \mu \mathrm{m}$. We carried out perforation experiments on the same size 3D $4 \mathrm{H}-\mathrm{SiC}$ detector, which can verify the simulation results in the near future.

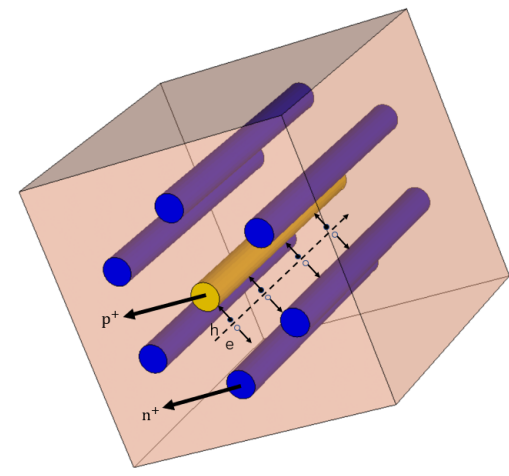

(a)

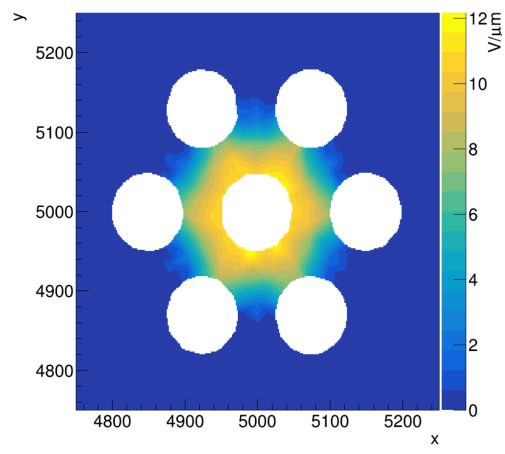

(b)

Figure 2. (a) 3D 4H-SiC detector schematic diagram. (b) The electric field distribution of $x-y$ cross section for the 3D 4H-SiC detector with $500 \mathrm{~V}$ bias voltage simulated with RASER.

The simulation of electric field in RASER is obtained by solving Poisson equations and Laplace equations [24] as shown in Figure 2b. The electric field in the central area between two adjacent $n^{+}$electrodes is weak, which will result in the time response of this part of the signal to be very slow. These signals will degrade the time-resolution performance and cause the distribution of the threshold time to be as Landau rather than Gaussian [18].

\subsection{Simulation of Induced Current}

The induced current is produced by the motion of the e-h pairs, generated on the tracks of charged particles passing through the detector shown as a dotted arrow in Figure $2 \mathrm{a}$. The magnitude of the induced current is mainly determined by the generated number of $\mathrm{e}-\mathrm{h}$ pairs and the magnitude of the electric field.

The number of $\mathrm{e}-\mathrm{h}$ pairs is determined by the deposited energy of charged particles. The $\beta$-setup as shown in Figure $3 \mathrm{a}$ is used to measure the time resolution of the $4 \mathrm{H}-\mathrm{SiC}$ 
detector. ${ }^{90}$ Sr source can emit two electrons with an energy of $0.55 \mathrm{MeV}$ and 2.28 MeV [27], which will deposit energy in the detector. The signals of the LGAD and 4H-SiC detectors induced by the same electron will be recorded by the oscilloscope after a total of 100 times amplification by the PCB board designed by the University of California Santa Cruz (UCSC) [28] and the main amplifier. The aluminum foil and the shield are mainly served to reduce the impact of the environmental background, and the time resolution of LGAD was 34 ps. We simulated two electrons' tracks and deposited energy by GEANT4 similar to the $\beta$-setup as shown in Figure 3b. The deposited energy in $350 \mu \mathrm{m}$ 3D detector is shown in Figure 3c, and the most probability value (MPV) was $0.14 \mathrm{MeV}$. The e-h pair creation energy of $4 \mathrm{H}-\mathrm{SiC}$ was $7.8 \mathrm{eV}$, and $51 \mathrm{e}-\mathrm{h}$ pairs $/ \mu \mathrm{m}$ were generated, which is consistent with the previous result of $56 \mathrm{e}-\mathrm{h}$ pairs $/ \mu \mathrm{m}$ [10].

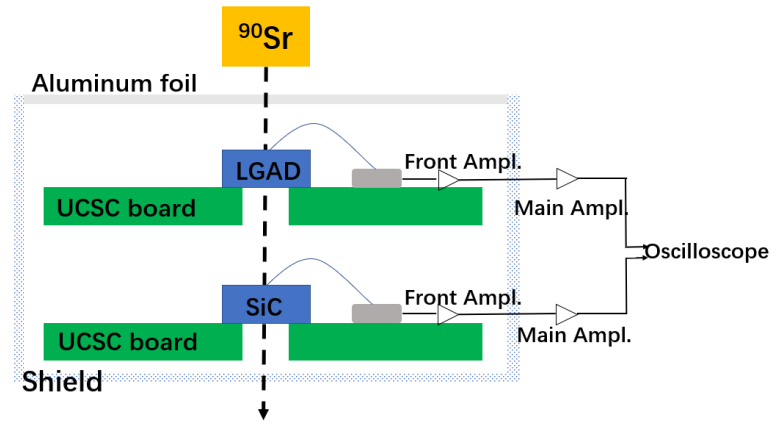

(a)

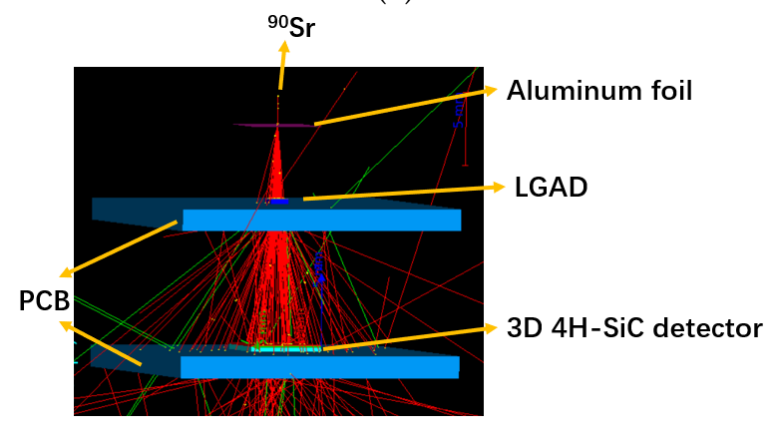

(b)

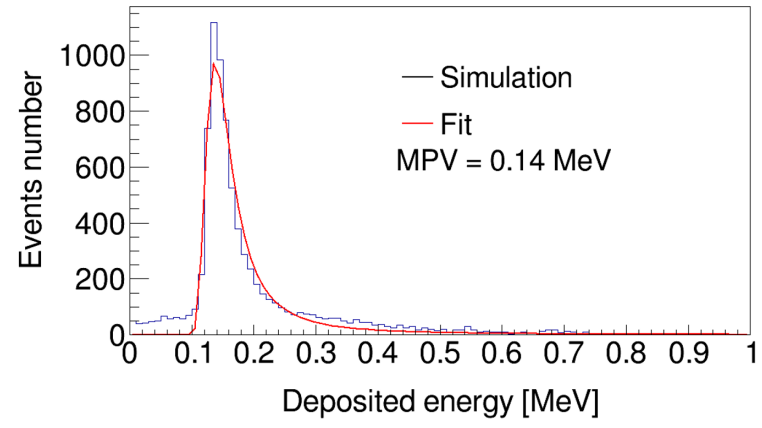

(c)

Figure 3. (Color online) (a) $\beta$-setup used to measure the time resolution of $4 \mathrm{H}$-SiC detector. (b) GEANT4 simulated two electrons passing through aluminum foil, the LGAD, a printed circuit board (PCB), and the 3D $4 \mathrm{H}-\mathrm{SiC}$ detector. The tracks of electrons and the deposited energy with a step less than $1 \mu \mathrm{m}$ were recorded. (c) Simulation of deposited energy distribution using 10,000 events in the 3D $4 \mathrm{H}-\mathrm{SiC}$ detector, and the MPV was $0.14 \mathrm{MeV}$. 
The instant-induced current by an electron or a hole can be calculated with the Shockley-Ramo's theorem [26]:

$$
I(t)=-q \vec{v}(\vec{r}(t)) \cdot \vec{E}_{w}(\vec{r}(t))
$$

where $\vec{r}$ is the drifting charge trajectory of electron or hole; q is the charge, $\vec{v}(\vec{r})$ is the drift velocity; and $\vec{E}_{w}(\vec{r})$ is the weighting potential. $\vec{v}(\vec{r})$ is calculated by $\mu_{S i C} \cdot E(\vec{r})$, and the mobility distribution of electron and hole in $4 \mathrm{H}-\mathrm{SiC}$ with electric field is shown in Figure 4a, where the mobility model refers to [29]. The velocity of the electron was $\sim 1.6 \times 10^{7} \mathrm{~cm} \cdot \mathrm{s}^{-1}$, and the hole was $\sim 9.5 \times 10^{6} \mathrm{~cm} \cdot \mathrm{s}^{-1}$ when the electric field in the detector was around $10 \mathrm{~V} / \mu \mathrm{m}$ as shown in Figure $4 \mathrm{~b}$. These velocities are close to the saturated electron or hole velocity at $300 \mathrm{~K}$, and the induced current will not change too much even if the voltage is raised.

The induced current is used as the input of readout electronics. The current induced by electron and hole moving is shown in Figure $4 c$, and the amplitude after electronics is shown as a pink line in Figure $4 \mathrm{~d}$. The maximum drift distance of the electron or the hole was $50 \mu \mathrm{m}$, which was calculated by the column spacing subtracted by twice the column radius, and the maximum drift time calculated by the drift-distance-divided velocity was about $0.6 \mathrm{~ns}$. This is consistent with the current simulation results.

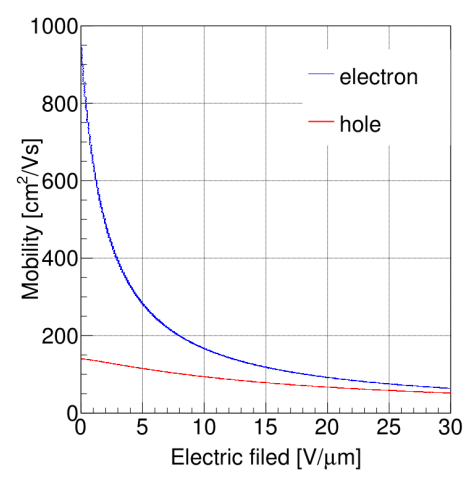

(a)

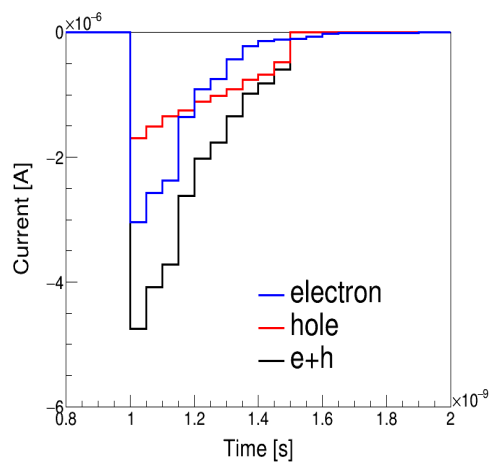

(c)

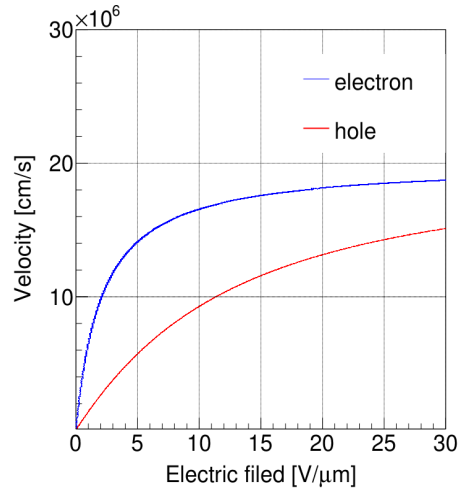

(b)

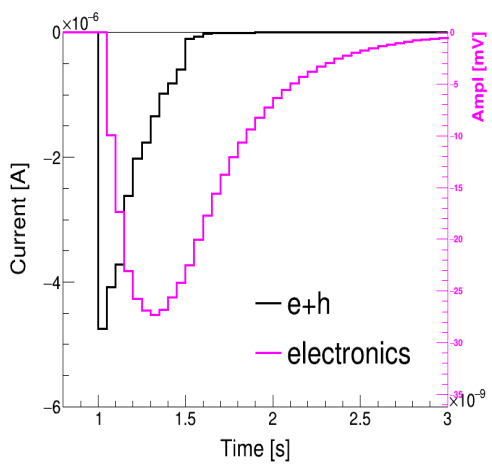

(d)

Figure 4. (Color online.) (a) The mobility model and (b) the velocity model of electron (blue) and hole (red) for $4 \mathrm{H}-\mathrm{SiC}$ used in RASER. (c) The distribution of induced current for electron (blue), hole (red), and electron plus hole (black). (d) The distribution of induced current for electron plus hole (black) and amplitude after electronics (pink). 


\section{Time-Resolution Simulation Results}

The time resolution $\sigma_{t}$ of the $4 \mathrm{H}-\mathrm{SiC}$ detector is mainly determined by jitter contribution $\sigma_{j i t t e r}$ and the time walk contribution $\sigma_{t w} . \sigma_{j i t t e r}$ can be calculated by the following equation [17]:

$$
\sigma_{\text {jitter }}=t_{p} /(S / N)
$$

where $t_{p}$ is the peak time of all waveforms, and $S / N$ is the signal-to-noise ratio. $\sigma_{j i t t e r}$ is mainly caused by electronics noise and the amplifier slew rate. We added measured electronics noise in RASER to simulate the contribution of jitter. $\sigma_{t w}$ is mainly caused by the difference in the signal height or the signal shapes. We used the constant fraction discrimination (CFD) method to minimize the effect of the signal height. The influences of the drift path and fluctuations in ionization rates on signal shapes were taken into account by GEANT4 as shown in Figure 3b,c.

We simulated the effects of different parameters such as temperature, voltage, and column spacing on the time resolution, as a reference for manufacturing 3D $4 \mathrm{H}-\mathrm{SiC}$ detectors. The time-resolution and rise-time changes with bias voltage are shown in Figure 5, where the rise time is obtained by fitting the distribution of the peak time for waveforms of all events with the Gaussian function. As indicated in Equation (2), the time resolution decreases, while the rise time decreases. When the voltage is less than $300 \mathrm{~V}$, the electric field in the detector and the velocity of $\mathrm{e}-\mathrm{h}$ pairs increase as the voltage increases. The velocity is much less than the saturated velocity, so the rise time will decrease. When the electric field is so high that the drift velocity is almost saturated, the drift velocity and the time resolution will not change by simply increasing the bias voltage.

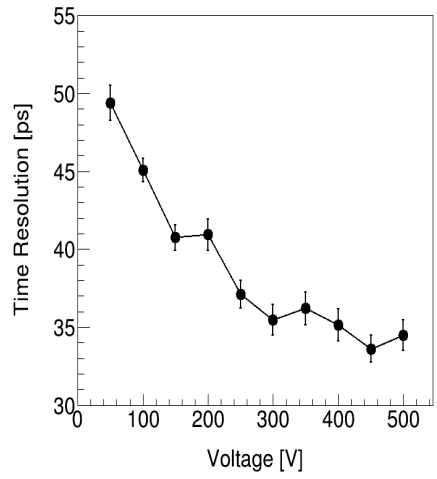

(a)

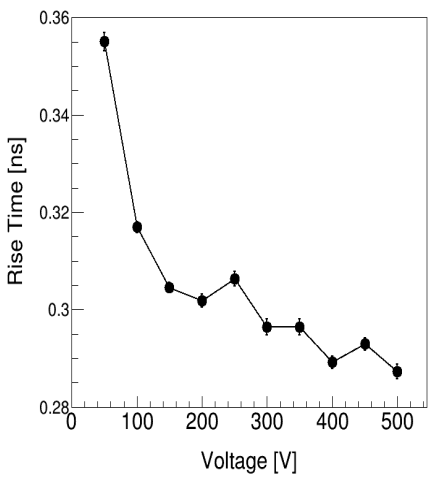

(b)

Figure 5. (a) Time resolution versus voltage. (b) Rise time versus voltage.

Figure 6a shows that temperature has little effect on the time resolution of the $4 \mathrm{H}-\mathrm{SiC}$ detector, even if the temperature reaches around $200{ }^{\circ} \mathrm{C}$. In the simulation, temperature only affects the mobility and therefore the time resolution, which may be inconsistent with the real situation. The $4 \mathrm{H}-\mathrm{SiC}$ has a lower dark current and associated noise than silicon because of its wide bandgap energy and low intrinsic carrier concentration. These mean the $4 \mathrm{H}-\mathrm{SiC}$ detector may work at higher temperatures than the silicon detector. As the temperature increases, the dark current increases. The dark current of the silicon $p-n$ junction usually limits the operating temperature of the silicon detector to no higher than $+30^{\circ} \mathrm{C}$, and the general working temperature is below $0{ }^{\circ} \mathrm{C}$. The $4 \mathrm{H}-\mathrm{SiC}$ detector can operate at $+127^{\circ} \mathrm{C}$ with the same current density as other detectors such as silicon and GaAs have at room temperature, which has been proved feasible by experiment [10]. The $4 \mathrm{H}-\mathrm{SiC}$ detector shows a good energy response for ${ }^{241} \mathrm{Am}$ at $200{ }^{\circ} \mathrm{C}$ after gamma-ray irradiation up to $1 \mathrm{MGy}$ in [30]. At the same time, the temperature of $4 \mathrm{H}-\mathrm{SiC}$ is easier to control due to its higher thermal conductivity. The 3D $4 \mathrm{H}-\mathrm{SiC}$ detector may operate at room temperature without a cooling system, which will greatly simplify the commission procedures. 
Figure $6 \mathrm{~b}$ shows the time resolution decreases as thickness increases. The reason is that the 3D $4 \mathrm{H}-\mathrm{SiC}$ detector decouples the thickness and column spacing. The rise time does not change with thickness, and pulse height increases as thickness increases, as shown in Figure $6 \mathrm{c}, \mathrm{d}$. The pulse height is the average of the highest amplitudes for all waveforms. The 3D 4H-SiC detector can increase the signal without increasing the rise time, which means a better signal-to-noise ratio, radiation hardness, and time resolution.

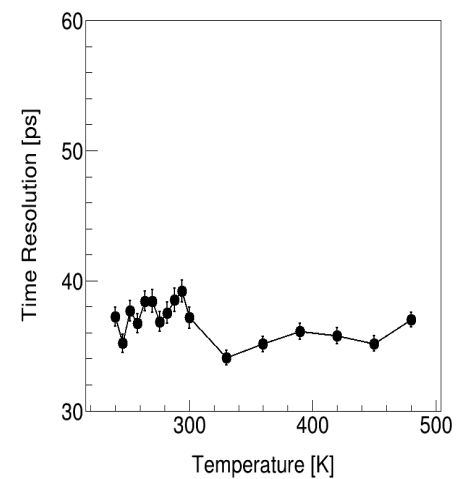

(a)

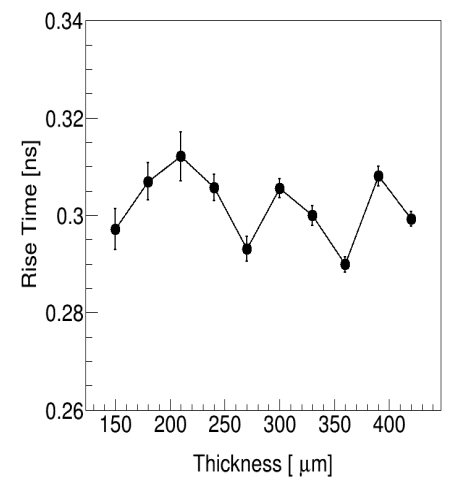

(c)

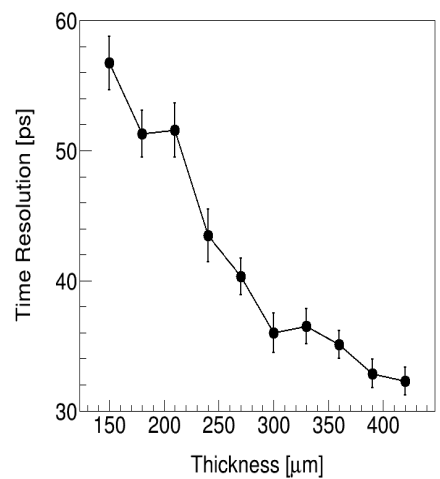

(b)

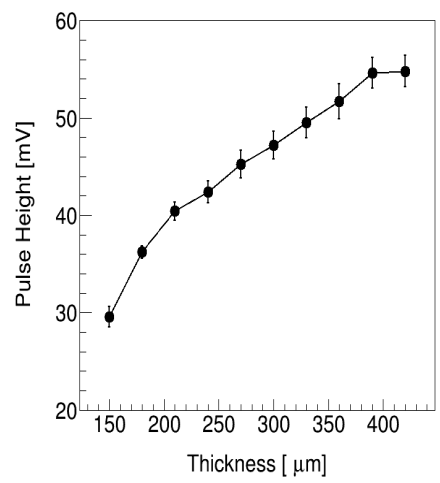

(d)

Figure 6. (a) Time resolution versus temperature. (b) Time resolution versus thickness. (c) Rise time versus thickness. (d) Pulse height versus thickness.

Figure 7a shows the distribution of $\sigma_{t w}, \sigma_{j i t t e r}$, and $\sigma_{t}$ with different voltages. $\sigma_{t}^{2} \approx \sigma_{t w}^{2}$ $+\sigma_{j i t t e r}^{2} . \sigma_{j i t t e r}$ decreases as the voltage increases in the range of $50 \mathrm{~V}$ to $150 \mathrm{~V}$, because an increase in the electric field will reduce $t_{p}$ and increase the signal-peak value in Equation (2). The change in voltage does not affect the hit position and deposited energy, so $\sigma_{t w}$ is almost unchanged. $\sigma_{t w}$ will increase with a column spacing increase as shown in Figure $7 \mathrm{~b}$, because the value of column spacing will change the hit-position area. Figure $7 \mathrm{~b}$ also shows that the increase in the column spacing increases the drift time and thus $\sigma_{j i t t e r}$. These results are consistent with expectations. 


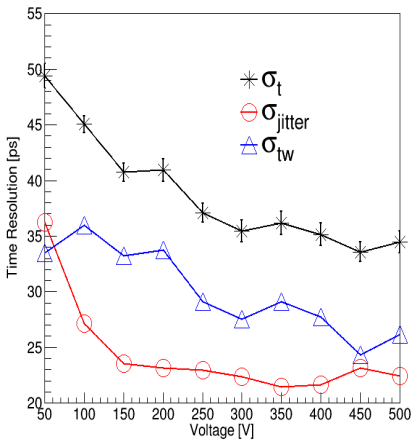

(a)

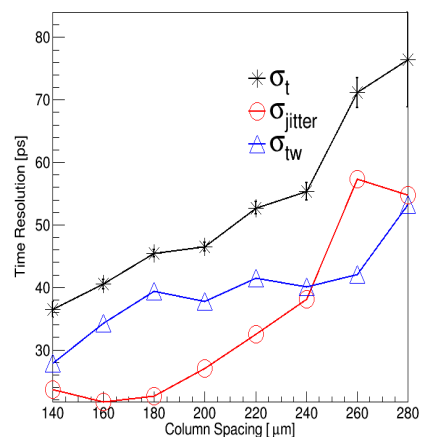

(b)

Figure 7. (Color online.) The distribution of $\sigma_{t w}, \sigma_{j i t t e r}$, and $\sigma_{t}$ with (a) different voltage and (b) different column spacing.

The simulation parameters and results of planar $4 \mathrm{H}-\mathrm{SiC}, 3 \mathrm{D} 4 \mathrm{H}-\mathrm{SiC}$-with-sevenelectrodes (3D-4H-SiC-7E), and 3D 4H-SiC-with-five-electrodes (3D-4H-SiC-5E) detectors are given in Table 1 . The electric field of the $\mathrm{x}-\mathrm{y}$ cross section for $3 \mathrm{D}-4 \mathrm{H}-\mathrm{SiC}-5 \mathrm{E}$ detector is shown in Figure 8, where the column spacing is $150 \mu \mathrm{m}$. The 3D-4H-SiC-5E detector removes two $n^{+}$electrodes based on the structure of Figure 2b, and the remaining four $n^{+}$ electrodes become a square distribution. The time resolution of the planar detector is larger than the 3D detector because of a smaller pulse height and a larger rise time. The time resolutions of 3D-4H-SiC-5E are slightly better than the 3D-4H-SiC-7E configuration. The pulse height of $3 \mathrm{D}-4 \mathrm{H}-\mathrm{SiC}-7 \mathrm{E}$ detector is smaller, because the more electrodes, the smaller the effective area. Table 1 reveals that the 3D detector can achieve a smaller rise time and a larger pulse height simultaneously, which means that the 3D detector has the potential to become a good time-resolution and a high-radiation-resistance detector. To achieve a better time resolution and the simplification of the fabrication process, 3D 4H-SiC detectors produced in the future will be mainly five electrodes.

Table 1. The simulation parameters and results for planar 4H-SiC, 3D-4H-SiC-7E, and 3D-4H-SiC-5E detectors with $500 \mathrm{~V}$ bias voltage.

\begin{tabular}{|c|c|c|c|c|c|}
\hline SiC Detector Type & Column Spacing ( $\mu \mathrm{m})$ & Thickness ( $\mu \mathrm{m})$ & Rise Time (ns) & Pulse Height (mV) & Time Resolution (ps) \\
\hline Planar & 100 & 100 & 0.38 & 13 & 77 \\
\hline 3D-4H-SiC-7E & 50 & 350 & 0.29 & 48 & 34 \\
\hline 3D-4H-SiC-5E & 50 & 350 & 0.32 & 53 & 25 \\
\hline
\end{tabular}

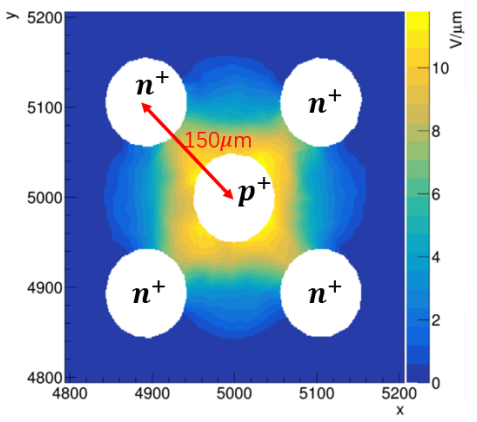

Figure 8. The electric field distribution of $x-y$ cross section for 3D-4H-SiC-5E detector with $500 \mathrm{~V}$ bias voltage.

\section{Conclusions}

The 3D 4H-SiC detector has the potential to achieve a good time resolution and high radiation resistance operating at room temperature to meet the challenges of future high- 
energy collider experiments. We developed a simulation program, RASER, to simulate the time resolution of the 3D $4 \mathrm{H}-\mathrm{SiC}$ detector with different structures and parameters. RASER was validated by comparing time-resolution measurement and simulation results of planar $4 \mathrm{H}-\mathrm{SiC}$ detectors, and the track simulation of two electrons from the ${ }^{90} \mathrm{Sr}$ and the mobility model were introduced. In addition, the advantage of the 3D $4 \mathrm{H}-\mathrm{SiC}$ detector is that it can work at room temperature and may not require a complicated cooling system.

The distribution of time resolution with different voltages, thicknesses, and column spacings were simulated, and the relationship between $\sigma_{t w}, \sigma_{j i t t e r}$, and $\sigma_{t}$ are also shown in Figure 7. The simulation results reveal that time resolution increases with a voltage decrease or a thickness decrease or a column-spacing increase, which were as expected. The time resolution of the $4 \mathrm{H}-\mathrm{SiC}$ detectors with two different numbers of electrodes was simulated, and it was found that the 3D-4H-SiC-5E configuration can reach up to a 25 ps time resolution, which is better than the $3 \mathrm{D}-4 \mathrm{H}-\mathrm{SiC}-7 \mathrm{E}$ configuration. These simulation results will be used as guideline for 3D $4 \mathrm{H}-\mathrm{SiC}$ detector design and will be verified in future 3D SiC devices.

Author Contributions: Conceptualization, X.S.; methodology, Y.T. and T.Y.; software, Y.T., Y.Z. and C.F.; validation, C.W., X.Z. (Xiyuan Zhang) and M.Z.; formal analysis, Y.T.; investigation, X.X. and R.X.; resources, X.S., X.X. and H.L.; data curation, T.Y. and Y.T.; writing-original draft preparation, Y.T.; writing-review and editing, X.S., K.L. and X.Z. (Xinbo Zou); visualization, Y.T.; supervision, Z.Z. and R.F.; project administration, X.S.; funding acquisition, X.S., W.S. and X.K. All authors have read and agreed to the published version of the manuscript.

Funding: This research was supported by the National Natural Science Foundation of China (No.11961141014, No. 12075045, No. 11905092, No. 12105132, and No. 12147217); the Foundation of Liaoning Province of China (No. 2019-BS-113); the scientific research fund project of the Liaoning Provincial Department of Education (No. LQN201902); the State Key Laboratory of Particle Detection and Electronics, China (SKLPDE-ZZ-202001); and the Program of Science and Technology Development Plan of Jilin Province of China under Contract No. 20210508047RQ.

Acknowledgments: This work was performed under the RD50 framework. We gratefully acknowledge the effort of the IHEP computing staff in providing us with an excellent computing system.

Conflicts of Interest: The authors declare no conflict of interest. The funders had no role in the design of the study; in the collection, analyses, or interpretation of data; in the writing of the manuscript, or in the decision to publish the results.

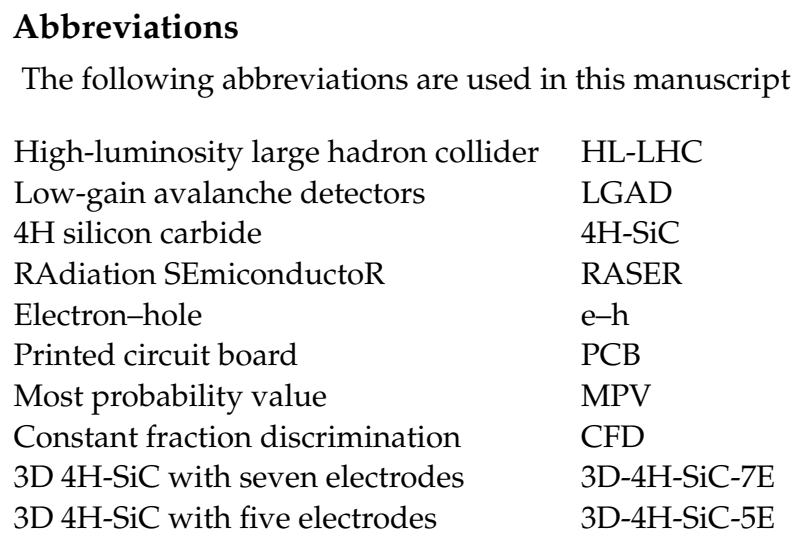

\section{References}

1. ATLAS Collaboration. Technical Design Report for the ATLAS Inner Tracker Strip Detector; Technical Report; CERN: Geneva, Switzerland, 2017. Available online: https://cds.cern.ch/record/2257755 (accessed on 29 November 2021).

2. Orfanelli, S. for the CMS collaboration. The Phase 2 Upgrade of the CMS Inner Tracker. Nucl. Instrum. Meth. A 2020, 980, 164396. [CrossRef]

3. Macchiolo, A. on behalf of the CMS Collaboration. The Phase-2 ATLAS ITk pixel upgrade. Nucl. Instrum. Meth. A 2020, 962, 162261. [CrossRef] 
4. ATLAS Collaboration. Technical Design Report: A High-Granularity Timing Detector for the ATLAS Phase-II Upgrade; Technical Report; CERN: Geneva, Switzerland, 2020. Available online: https://cds.cern.ch/record/2719855 (accessed on 29 November 2021).

5. Kramberger, G.; Baselga, M.; Cindro, V.; Fernandez-Martinez, P.; Flores, D.; Galloway, Z.; Gorišek, A.; Greco, V.; Hidalgo, S.; Fadeyev, V.; et al. Radiation effects in Low Gain Avalanche Detectors after hadron irradiations. JINST 2015, 10, P07006. [CrossRef]

6. Parker, S.I.; Kenney, C.J.; Segal, J. 3D-A proposed new architecture for solid-state radiation detectors. Nucl. Instrum. Meth. A 1997, 395, 328-343. [CrossRef]

7. Duarte-Campderros, J.; Curras, E.; Fernandez, M.; Gomez, G.; Garcia, A.; Gonzalez, J.; Silva, E.; Vila, I.; Jaramillo, R.; Meschini, M.; et al. Results on proton-irradiated 3D pixel sensors interconnected to RD53A readout ASIC. Nucl. Instrum. Meth. A 2019, 944, 162625. [CrossRef]

8. Werner, M.R.; Fahrner, W.R. Review on materials, microsensors, systems and devices for high-temperature and harsh-environment applications. IEEE Trans. Ind. Electron. 2001, 48, 249-257. [CrossRef]

9. Sellin, P.J.; Vaitkus, J. New materials for radiation hard semiconductor dectectors. Nucl. Instrum. Meth. A 2006, 557, 479-489. [CrossRef]

10. Nava, F.; Bertuccio, G.; Cavallini, A.; Vitton, E. Silicon carbide and its use as a radiation detector material. IOP Publ. 2008, $19,102001$.

11. DaVia, C.; Watts, S.J. The geometrical dependence of radiation hardness in planar and 3D silicon detectors. Nucl. Instrum. Meth. A 2009, 603, 319-324. [CrossRef]

12. Capeans, M.; Darbo, G.; Einsweiller, K.; Elsing, M.; Flick, T.; Garcia-Sciveres, M.; Gemme, C.; Pernegger, H.; Rohne, O.; Vuillermet, R. ATLAS Insertable B-Layer Technical Design Report; Technical Report; CERN: Geneva, Switzerland, 2010. Available online: https:/ / cds.cern.ch/record/1291633 (accessed on 29 November 2021).

13. Albrow, M.; Arneodo, M.; Avati, V.; Baechler, J.; Cartiglia, N.; Deile, M.; Gallinaro, M.; Hollar, J.; Lo Vetere, M.; CMS-TOTEM, Collaboration. CMS-TOTEM Precision Proton Spectrometer; Technical Report; CERN: Geneva, Switzerland, 2014. Available online: https:/ / cds.cern.ch/record/1753795 (accessed on 29 November 2021).

14. Kohler, M.; Bates, R.; Fleta, C.; Jakobs, K.; Lozano, M.; Parkes, C.; Parzefall, U.; Pellegrini, G.; Preiss, J. Comparative measurements of highly irradiated n-in-p and p-in-n 3D silicon strip detectors. Nucl. Instrum. Meth. A 2011, 659, 272-281. [CrossRef]

15. Rafi, J.M.; Joan, M.; Pellegrini, G.; Godignon, P.; Ugobono, S.O.; Rius, G.; Tsunoda, I.; Yoneoka, M.; Takakura, K.; Kramberger, G.; et al. Electron, Neutron, and Proton Irradiation Effects on SiC Radiation Detectors. IEEE Trans. Nucl. Sci. 2020, 67, 2481-2489. [CrossRef]

16. Bruzzi, M.; Adey, J.; Al-Ajili, A.; Alexandrov, P.; Alfieri, G.; Allport, P.P.; Andreazza, A.; Artuso, M.; Assouak, S.; Avset, B.S.; et al. Radiation-hard semiconductor detectors for SuperLHC. Nucl. Instrum. Meth. A 2005, 541, 189-201. [CrossRef]

17. Kramberger, G.; Cindro, V.; Flores, D.; Hidalgo, S.; Hiti, B.; Manna, M.; Mandić, I.; Mikuž, M.; Quirion, D.; Pellegrini, G.; et al Timing performance of small cell 3D silicon detectors. Nucl. Instrum. Meth. A 2019, 934, 26-32. [CrossRef]

18. Lai, A.; on behalf of the TIMESPOT Project and Team. Timing characterisation of 3D-trench silicon sensors. JINST 2020, 15, C09054. [CrossRef]

19. KDetSim. Available online: http:/ / kdetsim.org (accessed on 1 February 2021).

20. Cenna, F.; Cartiglia, N.; Friedl, M.; Kolbinger, B.; Sadrozinski, H.F.-W.; Seiden, A.; Zatserklyaniy, A.; Zatserklyaniy, A. Weightfield2: A fast simulator for silicon and diamond solid state detector. Nucl. Instrum. Meth. A 2015, 796, 149-153. [CrossRef]

21. RASER. Available online: https://pypi.org/project/raser (accessed on 1 November 2021).

22. Alnaes, M.S.; Blechta, J.; Hake, J.; Johansson, A.; Kehlet, B.; Logg, A.; Richardson, C.; Ring, J.; Rognes, M.E.; Wells, G.N. The FEniCS Project Version 1.5. Arch. Numer. Softw. 2015, 3, 9-23.

23. Fan, Y.Y.; Alderweireldt, S.; Agapopoulou, C.; Atanov, N.; Kassem Ayoub, M.; Caforio, D.; Chen, H.; Christie, S.; da Costa, J.G.; Cui H.; et al. Radiation hardness of the low gain avalanche diodes developed by NDL and IHEP in China. Nucl. Instrum. Meth. A 2020, 984, 164608. [CrossRef]

24. Yang, T.; Tan, Y.H.; Liu, Q.; Xiao, S.Y.; Liu, K.; Zhang, J.Y.; Kiuchi, R.; Zhao, M.; Wu, B.Y.; Lin, J.N.; et al. Time resolution of 4H-SiC PIN detector. arXiv 2021, arXiv:2105.15068.

25. GEANT4. Available online: https://geant4.web.cern.ch (accessed on 1 July 2021).

26. Ramo, S. Currents induced by electron motion. Proc. Ire. 1939, 27, 584-585. [CrossRef]

27. Ferrero, M.; Arcidiacono, R.; Mandurrino, M.; Sola, V.; Cartiglia, N. An Introduction to Ultra-Fast Silicon Detectors; Publishing House: Boca Raton, FL, USA, 2021; p. 196.

28. Galloway, Z.; Fadeyev, V.; Freeman, P.; Gkougkousis, E.; Gee, C.; Gruey, B.; Labitan, C.A.; Luce, Z.; McKinney-Martinez, F.; Sadrozinski, H.F.-W.; et al. Properties of HPK UFSD after neutron irradiation up to $6 \mathrm{e} 15 \mathrm{n} / \mathrm{cm}^{2}$. Nucl. Instrum. Meth. A 2019, 940 , 19-29. [CrossRef]

29. Das, A.; Duttagupta, S.P. TCAD simulation for alpha-particle spectroscopy using SIC Schottky diode. Radiat. Prot. Dosim. 2015, 167, 443-452. [CrossRef] [PubMed]

30. Li, Z.; Wu, J.; Wu, K.1.; Fan, Y.K.; Bai, Z.X.; Jiang, Y.; Yin, Y.P.; Xie, Q.L.; Lei, J.R. The performance of 4H-SiC detector at high temperature after gamma irradiation. Radiat. Phys. Chem. 2019, 162, 153-156. [CrossRef] 